\title{
On the role of neutral flow in field-aligned currents
}

\author{
Anthony J. Mannucci ${ }^{1}$, Olga P. Verkhoglyadova ${ }^{1}$, Xing Meng ${ }^{1}$, and Ryan McGranaghan ${ }^{1,2}$ \\ ${ }^{1}$ Jet Propulsion Laboratory, California Institute of Technology, Pasadena, CA 91109, USA \\ ${ }^{2}$ University Corporation for Atmospheric Research (UCAR), CPAESS, Boulder, CO 80301, USA
}

Correspondence: Anthony J. Mannucci (anthony.j.mannucci@jpl.nasa.gov)

Received: 27 May 2017 - Revised: 11 October 2017 - Accepted: 27 November 2017 - Published: 18 January 2018

\begin{abstract}
In this brief note we explore the role of the neutral atmosphere in magnetosphere-ionosphere coupling. We analyze momentum balance in the ion rest frame to form hypotheses regarding the role of neutral momentum in the lower ionosphere during geomagnetic storms. Neutral momentum that appears in the ion rest frame is likely the result of momentum imparted to ionospheric ions by solar wind flow and the resultant magnetospheric dynamics. The resulting ionneutral collisions lead to the existence of an electric field. Horizontal electron flow balances the momentum supplied by this electric field. We suggest a possible role played by the neutral atmosphere in generating field-aligned currents due to local auroral heating. Our physical interpretation suggests that thermospheric neutral dynamics plays a complementary role to the high-latitude field-aligned currents and electric fields resulting from magnetospheric dynamics.
\end{abstract}

Keywords. Ionosphere (ionosphere-magnetosphere interactions; polar ionosphere) - magnetospheric physics (magnetosphere-ionosphere interactions)

\section{Introduction}

Momentum transfer from the solar wind to the magnetosphere and ionosphere is a significant source of geospace dynamics, which is enhanced during periods of geomagnetic activity. A number of consequences are known to occur, such as increased field-aligned currents (FACs) that flow between the ionosphere and magnetosphere, enhanced ionization due to precipitating particles, increased and enlarged high-latitude plasma convection, and neutral atmosphere heating (Kelley, 2009; Brekke, 2013). The complex physical processes that occur in the partially ionized, collisional plasma that resides in the lower ionosphere are often described using Ohm's law (Song et al., 2001), which is a relationship between electric and magnetic fields and currents in the plasma. Ohm's law can be derived based on the quasi-static assumption that electric, magnetic, and collisional forces within the plasma are balanced (Vasyliunas, 2012). Although Ohm's law is less applicable during highly dynamic situations in which changes in plasma inertia play a role, it is nevertheless widely used to understand electrodynamic relationships between different elements of the plasma and interactions with the neutral species in which the plasma is embedded.

In this paper, we analyze the plasma momentum balance equations in the ion rest frame in order to gain insight into cause and effect relationships in the high-latitude ionosphere, specifically with respect to magnetosphere-ionosphere coupling. In this frame we deduce the importance of the solar wind in imparting momentum to the neutral thermosphere through the intermediary of magnetosphere dynamics, and we discuss the implications. The analysis occurs in the ion rest frame because of certain clarifying features of this frame.

\section{Momentum balance equations}

Song et al. (2001) (hereafter Song01) derived a three-fluid version of Ohm's law to deduce relationships between currents and electric and magnetic fields taking collisions with neutral species into account. The starting point is the momentum balance equations governing ions, electrons, and neutrals, assuming charge neutrality (Eqs. 4 and 5 in Song01; see also Brekke and Rino, 1978):

$$
\begin{aligned}
q N_{\mathrm{e}}\left(\boldsymbol{E}+\boldsymbol{u}_{\mathrm{i}} \times \boldsymbol{B}\right)= & N_{\mathrm{e}} m_{\mathrm{i}} v_{\mathrm{in}}\left(\boldsymbol{u}_{\mathrm{i}}-\boldsymbol{u}_{\mathrm{n}}\right) \\
& +N_{\mathrm{e}} m_{\mathrm{i}} v_{\mathrm{ie}}\left(\boldsymbol{u}_{\mathrm{i}}-\boldsymbol{u}_{\mathrm{e}}\right), \\
-q N_{\mathrm{e}}\left(\boldsymbol{E}+\boldsymbol{u}_{\mathrm{e}} \times \boldsymbol{B}\right)= & N_{\mathrm{e}} m_{\mathrm{e}} v_{\mathrm{en}}\left(\boldsymbol{u}_{\mathrm{e}}-\boldsymbol{u}_{\mathrm{n}}\right) \\
& -N_{\mathrm{e}} m_{\mathrm{e}} v_{\mathrm{ei}}\left(\boldsymbol{u}_{\mathrm{i}}-\boldsymbol{u}_{\mathrm{e}}\right),
\end{aligned}
$$


where $q$ is the electron charge, $N_{\mathrm{e}}$ is the charge density of either electrons or ions, $\boldsymbol{E}$ is the electric field, $\boldsymbol{B}$ is the magnetic field, $m_{\mathrm{i}}$ and $m_{\mathrm{e}}$ are the ion and electron masses, respectively, and $\boldsymbol{u}_{\mathrm{e}}, \boldsymbol{u}_{\mathrm{n}}$, and $\boldsymbol{u}_{\mathrm{i}}$ are the electron, neutral, and ion velocities, respectively; $v_{\mathrm{in}}, v_{\mathrm{ie}}, v_{\mathrm{en}}$, and $v_{\mathrm{ei}}$ are the ionneutral, ion-electron, electron-neutral, and electron-ion momentum transfer rates (also called "collision rates"), respectively. We note that the following relation applies to all of these rates: $m_{k} v_{k l}=m_{l} v_{l k}$, where $k, l$ represents one of the species: electron, ion, or neutral (Gombosi, 2004), and $k \neq l$. Similarly to Song01, we assume that ionized oxygen is the dominant ion and ignore the others, although this choice does not affect our conclusions significantly. As in Song01, we also assume that atomic oxygen is the dominant neutral species at altitudes at which ionospheric conductivities are largest ( $\sim 110 \mathrm{~km}$; Kelley, 2009). Different relative concentrations of neutral species will change the numeric values pertinent to Eqs. (1) and (2) somewhat, but will not alter our overall conclusions. Charge neutrality is assumed throughout $\left(N_{\mathrm{e}} \approx N_{i}\right)$ where $N_{i}$ is the ion density. Pressure gradients are neglected in Eqs. (1) and (2) (see Eqs. 1 and 2 of Yokoyama et al., 2004, for inclusion of pressure effects).

We gain insight into high-latitude magnetosphereionosphere coupling by examining Eq. (1) in the ion rest frame, which has several advantages. We know that in the limit of no collisions and in the absence of pressure gradient forces, the electric field in the ion rest frame is zero (Bellan, 2008). Thus, we can reasonably conjecture that the presence of an electric field in the ion rest frame is intimately tied to collisions with neutrals.

Equations (1) and (2) reflect momentum balance and do not describe dynamics or cause and effect relationships (Richmond and Thayer, 2000). For example, the origin of the electric field is not specified. The equations merely express that, under quasi-static conditions, electromagnetic forces are balanced by collisional forces. We will suggest below that collisions between ions and neutrals cause the electric field. A disadvantage of using the ion rest frame is that we cannot, in this frame, analyze cause and effect as it relates to ion motion, which is always zero. However, we understand that the primary cause of ion motion in the Earth rest frame is momentum supplied by the solar wind flowing past Earth.

In the following we assume that $\boldsymbol{B}$ in Eqs. (1) and (2) is the geomagnetic main field. Geomagnetic perturbations, being less than $1 \%$ of the main field, do not contribute significantly to quasi-static momentum balance considerations. Deviations, if any, from Earth's geomagnetic field are not retrievable from Eqs. (1) and (2) alone. However, deviations from or perturbations to the geomagnetic main field are essential elements of theories of inductive magnetosphereionosphere-thermosphere coupling, which require solutions to Maxwell's equations (Tu et al., 2014; Tu and Song, 2016).

Equations (1) and (2) have many solutions in terms of the variables $\boldsymbol{E}, \boldsymbol{u}_{\mathrm{e}}, \boldsymbol{u}_{\mathrm{n}}$, and $\boldsymbol{u}_{\mathrm{i}}$. A trivial and impractical solution to the above equations is that all velocities are zero and there is no momentum in the ion rest frame. This solution is not of interest during conditions leading to geomagnetic storms. The solar wind imparts momentum to ionospheric ions via electrodynamic forces, as is well known from observations of ion convection anti-sunward over the polar cap (Milan, 2017). The neutral species will be influenced by ion-neutral collisions and other forces, such as gravity and pressure forces due to coupling from below, and in general neutrals will be at a different velocity than the ions. We know from observations that frictional heating between ions and neutrals is a very prominent feature of the high-latitude ionosphere, particularly during storms (Thayer and Semeter, 2004; Kelley, 2009). This implies a velocity difference between ions and neutrals (Ridley et al., 2006). So, the trivial solution is too narrow to consider further, and there are clearly other important solutions for which $\boldsymbol{u}_{\mathrm{n}} \neq \boldsymbol{u}_{\mathrm{e}} \neq \mathbf{0}$ in the ion rest frame.

In the ion rest frame, Eqs. (1) and (2) become

$$
\begin{aligned}
& q \boldsymbol{E}=-m_{\mathrm{i}} v_{\mathrm{in}}\left(\boldsymbol{u}_{\mathrm{n}}\right)-m_{\mathrm{i}} v_{\mathrm{ie}}\left(\boldsymbol{u}_{\mathrm{e}}\right), \\
& -q\left(\boldsymbol{E}+\boldsymbol{u}_{\mathrm{e}} \times \boldsymbol{B}\right)=m_{\mathrm{e}} v_{\mathrm{en}}\left(\boldsymbol{u}_{\mathrm{e}}-\boldsymbol{u}_{\mathrm{n}}\right)+m_{\mathrm{e}} v_{\mathrm{ei}}\left(\boldsymbol{u}_{\mathrm{e}}\right) .
\end{aligned}
$$

By focusing on the ion momentum Eq. (3) and putting in typical values for momentum transfer rates (e.g., Fig. 1 in Song01 for altitude $\sim 110 \mathrm{~km}$ ), we have

$\boldsymbol{E}=-1.64 \times 10^{-4} \boldsymbol{u}_{\mathrm{n}}-3.07 \times 10^{-9} \boldsymbol{u}_{\mathrm{e}}$,

where the electric field is in units of volts per meter. Assuming that electron velocities do not exceed neutral velocities by $\sim 5$ orders of magnitude (justified below), the electric field in the ion rest frame is directly related to neutral flow, and the horizontal flow of electrons (which carry the current in the ion rest frame and flow at altitudes near $110 \mathrm{~km}$ ) is not important to ion momentum balance. We believe it is plausible that FACs of magnetospheric origin carried by electrons, which close horizontally in the ionosphere, do not play a significant role in the creation of the electric field that exists in the ion rest frame. It is more likely that such an electric field is caused by differential motion between ions and electrons due to the different momentum transfer rates between ions and neutrals and between electrons and neutrals. The generation of electric fields by ion-neutral velocity differences has been widely explored in the literature (e.g., Prakash and Pandey, 1985; Liperovsky et al., 1997; Yokoyama et al., 2004).

Momentum balance equations do not provide information on the causes of the electric field. However, it is clear that currents in the ion rest frame carried by electrons contribute negligibly to ion momentum balance at altitudes near $110 \mathrm{~km}$. An electric field must exist in this frame if neutral species have a different velocity than the ions. Electric currents that may be flowing are not constrained by ion momentum balance considerations alone.

The electric field appearing in Eq. (5) must also be used in the electron momentum balance Eq. (4). At $110 \mathrm{~km}$ of altitude, we have (using collision rates from Fig. 1 of Song01, 
which apply to $75^{\circ}$ latitude and local noon conditions near solar minimum; 1976)

$$
\begin{aligned}
\boldsymbol{E}= & 8.14 \times 10^{-8}\left(\boldsymbol{u}_{\mathrm{e}}-\boldsymbol{u}_{\mathrm{n}}\right)-3.07 \times 10^{-9} \boldsymbol{u}_{\mathrm{e}} \\
& -5.0 \times 10^{-5} \boldsymbol{u}_{\mathrm{e}},
\end{aligned}
$$

where we have assumed a typical magnetic field value of $50000 \mathrm{nT}$ and a vertical orientation. In the E-region ionosphere, electric fields perpendicular to magnetic field lines are generally much larger than parallel electric fields (Kelley, 2009). Thus, momentum balance in Eq. (6) is achieved at high latitudes with horizontal electric fields and horizontal electron flow. If horizontal electron velocities are not orders of magnitude different than neutral velocities (first term in Eq. 6), electron momentum balance in the ion rest frame is largely achieved by the $u_{\mathrm{e}} B$ Lorentz force term (third term in Eq. 6). We discuss electron velocities in the ion rest frame below.

A reasonable physical interpretation is that, once a steadystate velocity difference is established between ions and neutrals, an electric field must exist in the ion rest frame and horizontal currents $\left(\boldsymbol{u}_{\mathrm{e}} \neq 0\right)$ will flow. Currents of magnetospheric origin, while they clearly exist, need not be invoked as a "driving" factor in the ionospheric response because they may contribute negligible horizontal momentum. The primary "driver" of high-latitude convection is momentum imparted to ionospheric plasma by the solar wind via magnetospheric convection, leading to ion-neutral velocity differences (Vasyliunas, 2001; Song et al., 2009; Tu et al., 2011).

The horizontal currents created as a result of magnetospheric dynamics will be consistent with FAC flowing where the horizontal currents diverge (Milan, 2013). The primary role of magnetospheric dynamics in MI coupling suggests that MHD wave coupling approaches, including the lowest frequencies, should be viewed as fundamental to understanding the coupled geospace system ( $\mathrm{Tu}$ et al., 2014; Tu and Song, 2016).

Our conclusions depend strongly on altitude (Richmond and Thayer, 2000). As altitude increases the ion-neutral collision rate drops rapidly (e.g from $900 \mathrm{~s}^{-1}$ at $110 \mathrm{~km}$ to $25 \mathrm{~s}^{-1}$ at $150 \mathrm{~km}$ of altitude), lowering the electric field in the ion rest frame. At these higher altitudes, the influence of the neutral motion on the electrodynamics is much smaller.

\section{Discussion}

In the above analysis, conducted in the ion rest frame and applicable to the lower ionosphere (i.e., altitudes less than $150 \mathrm{~km}$ ), we have deduced that currents coming into the ionosphere due to magnetospheric processes are not a significant source of horizontal momentum for the high-latitude ionosphere. Therefore, magnetospheric currents may not be causally responsible for horizontal flow in the ionosphere near altitudes of $\sim 110 \mathrm{~km}$ at which collisions with neutrals are important.

Our conclusions depend to some degree on an estimate of the values of the electron velocities. If the electron velocities were large enough, electron momentum could be a more significant factor than we have suggested in the above. We can estimate electron velocities from typical (Earthfixed) FAC current values (e.g., Iijima and Potemra, 1976) of $\sim 2 \mu \mathrm{Am}^{-2}$, leading to electron velocities of $\sim 2 /\left(q N_{\mathrm{e}}\right) \sim$ $156 \mathrm{~m} \mathrm{~s}^{-1}$, assuming typical electron density values of $8 \times$ $10^{10} \mathrm{el} \mathrm{m}^{-3}$ near $110 \mathrm{~km}$ of altitude. Electron velocities in this range do not supply sufficient momentum to alter the above conclusions. We note that currents are approximately independent of reference frame.

Although our hypothesis has been derived using the ion rest frame, cause and effect relationships are not dependent on this frame. Relative velocities (e.g., between ions and neutrals) are also independent of reference frame. Considering an Earth-fixed reference frame means ion motion can exist and contribute to currents, and electric fields increase overall relative to the electric fields in the ion rest frame. In the neutral wind reference frame, neutral momentum is zero and it might appear that electrodynamic processes in the magnetosphere are the only significant causal factors. In a neutral wind reference frame, no cause and effect relationship involving neutral momentum is revealed by momentum balance considerations. However, in the ion rest frame, momentum balance is strongly suggestive that the velocities of neutral species must play a role in FAC generation. Neutral winds in the plasma rest frame can arise from magnetospheric dynamics or from dynamic interactions involving only neutral species.

We can carry our hypothesis into the Earth-fixed reference frame (or any reference frame). The advantage of using the ion rest frame as a starting point is that we know in this frame that the electric field is zero if collisions are absent and pressure gradients are ignored. The origin of electric fields in the Earth-fixed or neutral wind reference frames is harder to ascertain.

The role of locally generated neutral wind divergence is insufficiently explored as a source of FACs flowing between the magnetosphere and ionosphere. Neutral wind divergence in association with localized heating due to auroral precipitation may play a significant role in localized FAC generation. Cooper and Conde (2006) report divergence values in a locally heated region (via modelling) that are 1-2 orders of magnitude larger than divergence estimates from largescale convective motion (e.g., Thayer and Killeen, 1991). Using the electric field derived from Eq. (5) and a typical Hall conductivity value of $5 \times 10^{-4}$ Siemens $\mathrm{m}^{-1}$ integrated over $20 \mathrm{~km}$ of altitude, speed divergences of $1.3 \times 10^{-3} \mathrm{~s}^{-1}$ are sufficient to create FAC magnitudes of $\sim 2 \mu \mathrm{Am}^{-2}$. Such divergence values are consistent with estimates of divergence due to auroral arc heating (Cooper and Conde, 2006). 


\section{Conclusions}

We discuss a perspective on ionospheric electrodynamics that emphasizes the importance of ion-neutral velocity differences within the high-latitude ionosphere (see also Tu et al., 2011, and Vasyliunas, 2012). To the extent that such velocity differences are not caused by currents originating with magnetospheric processes, this perspective de-emphasizes magnetospheric current systems as a primary causative factor in magnetosphere-ionosphere coupling (however, see Milan et al., 2017; Milan, 2013; Thayer and Semeter, 2004; Cowley, 2000; Kan, 1987). We are inferring cause and effect relationships based on momentum balance considerations, not considerations of what changes the inertia of the charged or neutral species. Momentum balance and Ohm's law are not sufficient to derive the causal chain. However, they are suggestive and useful for forming hypotheses.

Field-aligned currents originating with magnetospheric processes are certainly a factor in the dynamics of the high-latitude ionosphere (Vasyliunas, 1970; Robinson et al., 1987). However, solar wind momentum coupled via electrodynamic forces to the ionosphere is what causes the large horizontal momentum increases that occur in the ionosphere during geomagnetic storms, and FACs are a consequence of this momentum coupling (Coxon et al., 2014). The complexity of geomagnetic storms creates challenging conditions in which to distinguish cause and effect relationships (Mannucci et al., 2015). Analysis of momentum balance in the ion rest frame suggests that collisions between ions and neutrals leads to an electric field causing horizontal electron currents. Divergence of these currents should be considered in terms of the neutral species contribution to FACs.

Our focus is on altitudes at which ion-neutral collisions are important and ionospheric currents flow. From an Earth-fixed perspective, if ion momentum in the ionosphere is traceable back to the momentum input from the solar wind and less so from details of magnetospheric current processes, then solar wind-ionosphere coupling is an important and possibly dominant causal mechanism in ionospheric electrodynamics (Tu et al., 2014; Tu and Song, 2016). Neutral dynamics due to localized heating should be further investigated in terms of their impact on field-aligned currents flowing between the ionosphere and magnetosphere.

Data availability. The data used in this paper are available upon request from the corresponding author (anthony.j.mannucci@jpl.nasa.gov).

Competing interests. The authors declare that they have no conflict of interest.
Acknowledgements. We acknowledge useful discussions with William Lotko at Dartmouth College. This research was carried out at the Jet Propulsion Laboratory, California Institute of Technology under contract with NASA. The sponsorship of NASA Heliophysics is acknowledged.

The topical editor, Ana G. Elias, thanks two anonymous referees for help in evaluating this paper.

\section{References}

Bellan, P. M.: Fundamentals of Plasma Physics, Cambridge University Press, Cambridge, UK, 2008.

Brekke, A.: Physics of the Upper Polar Atmosphere, Springer, Berlin, Heidelberg, Germany, 2013.

Brekke, A. and Rino, C. L.: High-resolution altitude profiles of the auroral zone energy dissipation due to ionospheric currents, J. Geophys. Res., 83, 2517-2524, https://doi.org/10.1029/JA083iA06p02517, 1978.

Cooper, S. L. and Conde, M.: Origins of horizontal divergence in the auroral thermosphere: a modelling study, Geophys. Res. Lett., 33, 1839-1835, https://doi.org/10.1029/2006GL027601, 2006.

Cowley, S. W. H.: Tutorial: Magnetosphere-ionosphere interactions: a tutorial review, magnetospheric current systems, edited by: Shin-ichi Ohtani, Geoph. Monog. Series, 118, 91-105, https://doi.org/10.1029/GM118p0091, 2000.

Coxon, J. C., Milan, S. E., Clausen, L. B. N., Anderson, B. J., and Korth, H.: The magnitudes of the regions 1 and 2 Birkeland currents observed by AMPERE and their role in solar wind-magnetosphere-ionosphere coupling, J. Geophys. Res., 119, 9804-9815, https://doi.org/10.1002/2014JA020138, 2014.

Gombosi, T. I.: Physics of the Space Environment, Revised, Cambridge University Press, Cambridge, UK, 2004.

Iijima, T. and Potemra, T. A.: Field-aligned currents in the dayside cusp observed by Triad, J. Geophys. Res., 81, 5971-5979, https://doi.org/10.1029/ja081i034p05971, 1976.

Kan, J. R.: Generation of field-aligned currents in magnetosphereionosphere coupling in a MHD plasma, Planet. Space Sci., 35 , 903-912, https://doi.org/10.1016/0032-0633(87)90068-7, 1987.

Kelley, M.: The Earth's Ionosphere, 2nd ed., Academic Press, Burlington, MA, USA, 2009.

Liperovsky, V. A., Meister, C.-V., Schlegel, K., and Haldoupis, C.: Currents and turbulence in and near mid-latitude sporadic $E$ layers caused by strong acoustic impulses, Ann. Geophys., 15, 767-773, https://doi.org/10.1007/s00585-997-0767-x, 1997.

Mannucci, A. J., Tsurutani, B. T., Verkhoglyadova, O. P., and Meng, X.: On scientific inference in geophysics and the use of numerical simulations for scientific investigations, Earth and Space Science, 2, 359-367, https://doi.org/10.1002/2015EA000108, 2015.

Milan, S. E.: Modeling Birkeland currents in the expanding/contracting polar cap paradigm, J. Geophys. Res., 118, 55325542, https://doi.org/10.1002/jgra.50393, 2013.

Milan, S. E., Clausen, L. B. N., Coxon, J. C., Carter, J. A., Walach, M.-T., Laundal, K., Østgaard, N., Tenfjord, P., Reistad, J., Snekvik, K., Korth, H., and Anderson, B. J.: Overview of solar wind-magnetosphere-ionosphere-atmosphere coupling and the generation of magnetospheric currents, Space Sci. Rev., 1-27, https://doi.org/10.1007/s11214-017-0333-0, 2017. 
Prakash, S. and Pandey, R.: Generation of electric fields due to the gravity wave winds and their transmission to other ionospheric regions, J. Atmos. Terr. Phys., 47, 363-374, https://doi.org/10.1016/0021-9169(85)90016-9, 1985.

Richmond, A. D. and Thayer, J. P.: TUTORIAL: Ionospheric Electrodynamics: A Tutorial, Magnetospheric Current Systems, edited by: Shin-ichi Ohtani, Geoph. Monog. Series, 118, 131, https://doi.org/10.1029/GM118p0131, 2000.

Ridley, A. J., Deng, Y., and Tóth, G.: The global ionospherethermosphere model, J. Atmos. Sol.-Terr. Phy., 68, 839-864, https://doi.org/10.1016/j.jastp.2006.01.008, 2006.

Robinson, R. M., Vondrak, R. R., Miller, K., Dabbs, T., and Hardy, D.: On calculating ionospheric conductances from the flux and energy of precipitating electrons, J. Geophys. Res., 92, 2565-2569, https://doi.org/10.1029/JA092iA03p02565, 1987.

Song, P., Gombosi, T. I., and Ridley, A. J.: Threefluid Ohm's law, J. Geophys. Res., 106, 8149-8156, https://doi.org/10.1029/2000JA000423, 2001.

Song, P., Vasyliūnas, V. M., and Zhou, X. Z.: Magnetosphereionosphere/thermosphere coupling: self-consistent solutions for a one-dimensional stratified ionosphere in three-fluid theory, J. Geophys. Res., 114, A08213-11, https://doi.org/10.1029/2008JA013629, 2009.

Thayer, J. P. and Killeen, T. L.: Vorticity and divergence in the highlatitude upper thermosphere, Geophys. Res. Lett., 18, 11891192, https://doi.org/10.1029/91g101225, 1991.

Thayer, J. P. and Semeter, J.: The convergence of magnetospheric energy flux in the polar atmosphere, J. Atmos. Sol.-Terr. Phy., 66, 807-824, https://doi.org/10.1016/j.jastp.2004.01.035, 2004.
Tu, J. and Song, P.: A two-dimensional global simulation study of inductive-dynamic magnetosphere-ionosphere coupling, J. Geophys. Res., 121, 11, https://doi.org/10.1002/2016JA023393, 2016.

Tu, J., Song, P., and Vasyliūnas, V. M.: Ionosphere/thermosphere heating determined from dynamic magnetosphereionosphere/thermosphere coupling, J. Geophys. Res., 116, A09311-9, https://doi.org/10.1029/2011JA016620, 2011.

Tu, J., Song, P., and Vasyliūnas, V. M.: Inductivedynamic magnetosphere-ionosphere coupling via MHD waves, J. Geophys. Res., 119, 530-547, https://doi.org/10.1002/2013JA018982, 2014.

Vasyliūnas, V. M.: Mathematical models of magnetospheric convection and its coupling to the ionosphere, Particles and Field in the Magnetosphere, 17, 60-71, https://doi.org/10.1007/978-94-0103284-1_6, 1970.

Vasyliūnas, V. M.: Electric field and plasma flow: what drives what? Geophys. Res. Lett., 28, 2177-2180, https://doi.org/10.1029/2001GL013014, 2001.

Vasyliūnas, V. M.: The physical basis of ionospheric electrodynamics, Ann. Geophys., 30, 357-369, https://doi.org/10.5194/angeo30-357-2012, 2012

Yokoyama, T., Yamamoto, M., Fukao, S., and Cosgrove, R. B.: Three-dimensional simulation on generation of polarization electric field in the midlatitude E-region ionosphere, J. Geophys. Res., 109, A01309, https://doi.org/10.1029/2003JA010238, 2004. 